\title{
APPLICATION OF THE DIMER AND TETRAMER MODELS FOR INTERPRETATION OF THE IR SPECTRA OF TEA(TCNQ) 2
}

\author{
I. Olejniczak and A. Graja \\ Institute of Molecular Physics, Polish Academy of Sciences \\ Smoluchowskiego 17, 60-179 Poznan, Poland
}

(Received May 7, 1993; revised version August 10, 1993)

\begin{abstract}
An interpretation of the IR polarized spectra of the organic quasi-one-dimensional semiconductor triethylammonium (TCNQ) $)_{2}$, between 80 and $300 \mathrm{~K}$, is discussed in terms of the dimer and tetramer theories. It is concluded that the dimer model is acceptable only for describing high- $T$ phase of TEA(TCNQ) $)_{2}$. The tetramer model is more adequate for an interpretation of the IR spectra of the salt within the large temperature range. It is also shown that the phase transition influences distinctly neither vibronic (electron-molecular vibration coupled) modes nor conformity of the experimental spectra with calculated ones according to the dimer or tetramer theory.

PACS numbers: 33.10.Lb, 33.20.Ea, 33.90." h, 78.30.Jw
\end{abstract}

\section{Introduction}

This study is concerned with the ion-radical salt triethylammonium-tetracyanoquinodimethane (TEA(TCNQ) $)_{2}$ ), the material whose physical properties were quite extensively investigated in the past (see [1]). However, in spite of an abundance of various experimental data, the problem of the phase transition in the salt remains open. Similarly, the theoretical modeling of the spectra at various temperatures is not solved satisfactorily.

TEA(TCNQ $)_{2}$ has a tetramerized columnar structure with small deviations from a regular structure. First the TCNQ monomers interact strongly in pairs to form identical dimers, then the dimers interact weakly. Thus the crystal structure shows typical irregular tetradic stacking of the TCNQ molecules. TEA(TCNQ) ${ }_{2}$ single crystals undergo a first order phase transition at $220 \mathrm{~K}$, which was discussed in our previous paper [1].

The purpose of the paper [1] was to investigate the evolution of the polarized IR reflectivity within the phase transition region. We drew the following conclusions: TEA(TCNQ $)_{2}$ undergoes a semiconductor-semiconductor phase transition 
at about $220 \mathrm{~K}$, which occurs mainly in the cation sublattice; the bands assigned to the cation vibrations exhibit distinct changes whereas totally symmetric $\left(a_{\mathrm{g}}\right)$ modes of the anions, coupled with molecular vibrations of TCNQ molecules or clusters, show to be changed much smaller. It was also suggested that the dimer model is acceptable for the description of the high-temperature phase of TEA(TCNQ) ${ }_{2}$.

The aim of the present paper is the interpretation of the polarized IR spectra of TEA(TCNQ $)_{2}$ in terms of two fundamental models, describing the electron-molecular vibration coupling in a broad temperature range: the model of isolated dimers with one electron per dimer and the model of tetramers with two electrons per tetramer.

\section{Short recapitulation of the used models}

In a number of organic semiconductors the acceptor molecules of TCNQ are present as quasi-isolated dimers. The theory $[2,3]$ of the optical properties of those compounds predicts sharp peaks in the polarized optical reflectance spectrum as a consequence of a coupling of the radical electrons to the totally symmetric $\left(a_{\mathrm{g}}\right)$ internal modes of vibration. Each dimer is assumed to be described by the Hamiltonian $(\hbar=1)$

$$
\begin{aligned}
& H=H_{\mathrm{e}}+H_{\mathrm{v}}+\sum_{\alpha, i} g_{\alpha} n_{i} Q_{\alpha, i}-\boldsymbol{F} \cdot \boldsymbol{p}, \\
& H_{\mathrm{v}}=\sum_{\alpha, i} \frac{1}{4}\left(\dot{Q}_{\alpha, i}^{2}+Q_{\alpha, i}^{2}\right) \omega_{\alpha},
\end{aligned}
$$

where $H_{\mathrm{e}}$ and $H_{\mathrm{v}}$, respectively, describe the radical electrons and the symmetrical molecular vibrations of each TCNQ molecule being a moiety of the dimer, in the absence of vibronic coupling. The vibronic or electron-molecular vibration couplings are specified by the third term of Eq. (1), where $\left\{g_{\alpha}\right\}(\alpha=1,2, \ldots, 10)$ are the linear electron-molecular vibration coupling constants, $n_{i}$ is the electron occupation number operator for the site $i(i=1,2), Q_{\alpha, i}$ denotes the (dimensionless) normal mode coordinate corresponding to the monomer $i$, while $\omega_{\alpha, i}$ denotes its corresponding normal mode frequency. $\boldsymbol{F}$ denotes an externally applied electric field and $\boldsymbol{p}$ - the radical electron electric dipole moment of the dimer [4].

The monomers in a dimer were assumed to be identical, therefore the frequencies $\omega_{\alpha, i}$ were the same for the sites $i=1,2$. Consequently, the complex conductivity along the stack direction is [3]

$$
\begin{aligned}
\sigma(\omega) & =-\frac{2 \pi i \omega e^{2} a^{2} N_{\mathrm{d}}}{4 h}\left[\frac{\omega_{\mathrm{CT}}\left(\omega_{\mathrm{CT}}^{2}-\omega^{2}-\mathrm{i} \omega \Gamma_{\mathrm{CT}}\right)}{8 t^{2}}\right. \\
& \left.-\sum_{\alpha} \frac{g_{\alpha}^{2} \omega_{\alpha}}{\omega_{\alpha}^{2}-\omega^{2}-\mathrm{i} \omega \gamma_{\alpha}}\right]^{-1} .
\end{aligned}
$$

IIere $N_{\mathrm{d}}$ denotes the number of dimers per unit volume, $a$ is interplanar spacing in dimers. $\omega_{\mathrm{CT}}$ and $\Gamma_{\mathrm{CT}}$ are, respectively, the energy and phenomenological natural width of the CT excitation, $t$ is the iransfer integral in the dimer, while $\gamma_{\alpha}$ denotes the phenomenological natural wisth of the antisymmetric vibration mode $\alpha$ of the 
dimer (the symmetric vibrations of the dimer do not couple with the intradimer electron excitations).

The stack organization in TEA(TCNQ) ${ }_{2}$ is neither exactly dimeric nor tetrameric. This is why other models, in particular a model of quasi-isolated tetramers should be acceptable for interpretation of the polarized reflection spectra of the salt. The tetramer with two electrons has been calculated by Yartsev [5] in terms of extended Iubbard model parameters. The frequency dependent conductivity may be found as

$$
\begin{aligned}
\sigma(\omega) & =-\frac{2 \pi i \omega e^{2} N_{\mathrm{t}}}{h} \\
& \times \frac{\chi_{11} A^{2}+2 \chi_{12} A B+\chi_{22} B^{2}+\left(\chi_{12}^{2}-\chi_{11} \chi_{22}\right)\left(A^{2} D^{\prime \prime}+B^{2} D^{\prime}\right)}{1-\chi_{11} D^{\prime}-\chi_{22} D^{\prime \prime}+\left(\chi_{11} \chi_{22}-\chi_{12}^{2}\right) D^{\prime} D^{\prime \prime}}
\end{aligned}
$$

where

$$
D^{\prime}(\omega)=\sum_{\alpha} \frac{g_{\alpha}^{\prime 2} \omega_{\alpha}^{\prime}}{\omega_{\alpha}^{\prime 2}-\omega^{2}-\mathrm{i} \omega \gamma_{\alpha}}, \quad D^{\prime \prime}(\omega)=\sum_{\alpha} \frac{g_{\alpha}^{\prime \prime 2} \omega_{\alpha}^{\prime \prime}}{\omega_{\alpha}^{\prime \prime 2}-\omega^{2}-\mathrm{i} \omega \gamma_{\alpha}} .
$$

IIere $N_{\mathrm{t}}$ is the number of tetramers per unit volume, $\gamma_{\alpha}$ denotes the natural width of the originally uncoupled vibrational modes, $A$ and $B$ are parameters describing the tetramer geometry and $\chi_{i j}$ are the reduced CT electronic polarizabilities.

It is very important that the $\sigma(\omega)$ of the tetramerized systems is found to produce a number of sharp maxima due to indirect excitation of the $a_{\mathrm{g}}$ internal molecular modes of vibrations. These peaks are shown to have a fine structure if the charge density is not shared equally by the constituent molecules [5]. The fine structure of the activated $a_{\mathrm{g}}$ modes was observed experimentally in TEA(TCNQ) $[6-8,1]$.

On the basis of structural data one can expect the dimer as well as the tetramer model to be adequate for interpreting the IR reflectivity of TEA(TCNQ) ${ }_{2}$. The dimerization of TCNQ columns, resulting from the presence of two different molecular overlappings and different interplanar distances, is an intrinsic property of this material, and the parameters of dimerization do not exhibit any significant modification between 40 and $345 \mathrm{~K}$ [9]. On the other hand, the unpaired electron is not shared equally by the two monomer sites [10], the difference between fractional charges being insignificant at high temperatures but increasing with lowering temperature. The crystal packing study of TEA(TCNQ) 2 shows simultaneously that the various interplanar spacings decrease with temperature, each to a different extent. The data extrapolated down to $0 \mathrm{~K}$ indicate that, at this temperature, each column is a pile of tetrads [10]. The dependence of totally symmetric $\left(a_{\mathrm{g}}\right)$ vibrations of the TCNQ molecule on its charge [11] leads to fine structure doublets becoming more pronounced with the decrease of temperature [1]. Considering its assumptions, the dimer model neglects the doublet structure of the activated $a_{\mathrm{g}}$ modes and, thus, one can anticipate rather good agreement with the experiment for TEA(TCNQ) 2 at high temperatures. On the contrary, the tetramer model, taking into consideration nonhomogeneous charge distribution, gives the doublet structure and should better describe the spectra of TEA(TCNQ) $)_{2}$ in the whole tcmperature range, in particular at low temperature. 
In the past a theoretical dimer fit to $300 \mathrm{~K}$ experimental data of TEA(TCNQ) 2 was made by Steigmeier at al. [7]. The natural widths of $a_{\mathrm{g}}$ modes $\gamma_{\alpha}$ were fixed as almost the same for each mode. Contrary to that approach, as the other authors' simplification relative to $\gamma_{\alpha}$ does not seem to be substantial, we attempted to fit natural widths $\gamma_{\alpha}$ as well. The tetramer model was tested by Yartsev [5] for the experimental data of Belousov et al. [8] at $300 \mathrm{~K}$ only.

\section{Experimental and data elaboration}

We measured the polarized reflection spectra of TEA(TCNQ) ${ }_{2}$ single crystals within the range $5000-400 \mathrm{~cm}^{-1}$ and at temperatures from 300 to $80 \mathrm{~K}$. The frequency dependent conductivity $\sigma(\omega)$ was calculated from the spectra, using the Kramers-Kronig analysis and two ranges of extrapolations, on the basis of Brau et al. [6] room temperature measurements, namely $50000-5000 \mathrm{~cm}^{-1}$ and $550-20 \mathrm{~cm}^{-1}$. A full description of the experiment was published in our previous paper [1].

The models with one radical electron per TCNQ dimer (formula (3)) and with two electrons per TCNQ tetramer (formula (4)) were used over the whole temperature range. The interplanar distances between the dimer moieties and numbers of dimers per $\AA^{3}$ were found from the structural data [10]. The transfer integrals were calculated according to Ref. [12] at all temperatures. The fitting was made between 4000 and $20 \mathrm{~cm}^{-1}$, using the Nelder-Mead simplex algorithm for minimizing a nonlinear function of several variables. The fitted parameters were the energy and natural width of the CT excitation $\omega_{\mathrm{CT}}$ and $\Gamma_{\mathrm{CT}}$, respectively, as well as the linear electron-molecular vibration coupling constants and natural widths of the $a_{\mathrm{g}}$ modes $\left\{g_{\alpha}, \gamma_{\alpha}\right\},(\alpha=2, \ldots, 10)$ except for a fixed value assumed for $\gamma_{6}\left(\omega_{6}=948 \mathrm{~cm}^{-1}\right)$. For one spectrum the fitting proceeded in several steps in order to a void too many fitted parameters in one go. Preliminary starting parameters were chosen in agreement with Steigmeier et al. [7]. First the spectrum for the lowest temperature was fitted; for successive temperatures, the fit obtained for a direct lower $T$ determined the starting set of parameter values.

The dimer model leaves out the doublet structure of the activated $a_{\mathrm{g}}$ modes and this disagreement is the main source of errors. However, first of all it affects the natural widths of the $a_{\mathrm{g}}$ modes $\gamma_{\alpha}$. The coupling constants $g_{\alpha}$ were calculated with an accuracy dependent on the mode $\alpha$. The essential source of errors, which are difficult to estimate, results from the choice of the theoretical model and the fitting procedure. It refers particularly to the range of $T$ below the phase transition temperature. In the case of the tetramer model, the fitting procedure was very close to that described above for the dimer model. It was assumed that the natural widths of both components of the vibronic bands are the same. We chose $U / V$ equal to 0.4 as assumed by Yartsev [5] for TEA(TCNQ) ${ }_{2}$ and $U=0.6 \mathrm{eV}$. Then using the matrix elements from the same paper we obtain the optimal values of $\Gamma_{\mathrm{CT}}, \omega_{\alpha}{ }^{\prime}, \omega_{\alpha}{ }^{\prime \prime}, \gamma_{\alpha}{ }^{\prime}=\gamma_{\alpha}{ }^{\prime \prime}, g_{\alpha}{ }^{\prime}$ and $g_{\alpha}{ }^{\prime \prime}$, where the former parameters relate to the main (wide) components of the doublets and the latter - to the narrow components.

The tetramer model describes the doublet structure of the activated $a_{\mathrm{g}}$ 
modes. The structure is very distinct in particular at low temperature. The quite good modeling of the doublet structure is the main advantage of the tetramer theory.

\section{Results and discussion}

The IR reflection spectra of the oriented TEA(TCNQ) 2 crystals, measured in polarized light at temperatures from 80 to $300 \mathrm{~K}$, were subjected to a Kramers-Kronig analysis. The obtained spectra of frequency dependent conductivity are dominated by a strong and very broad CT band at about $2300 \mathrm{~cm}^{-1}$ and a set of strong bands resulting from a vibronic activation of totally symmetric $a_{\mathrm{g}}$ modes of TCNQ, normally IR non-active. These bands are doublets with wide and narrow vibronic components; the fine structure is resolved particularly well in the lower temperature spectra [1].

It is useful to study the temperature changes of the $a_{\mathrm{g}}$ modes in terms of a model describing the electron-molecular vibration coupling. For a start, we applied the dimer model. Preliminary results were published in our previous paper [1]. The dimer theory provides a good general fit to the high-temperature data (Fig. 1); the calculated values of the frequencies and conductivities of the activated $a_{\mathrm{g}}$ modes are in good agreement with the experimental data. There was quite a good general

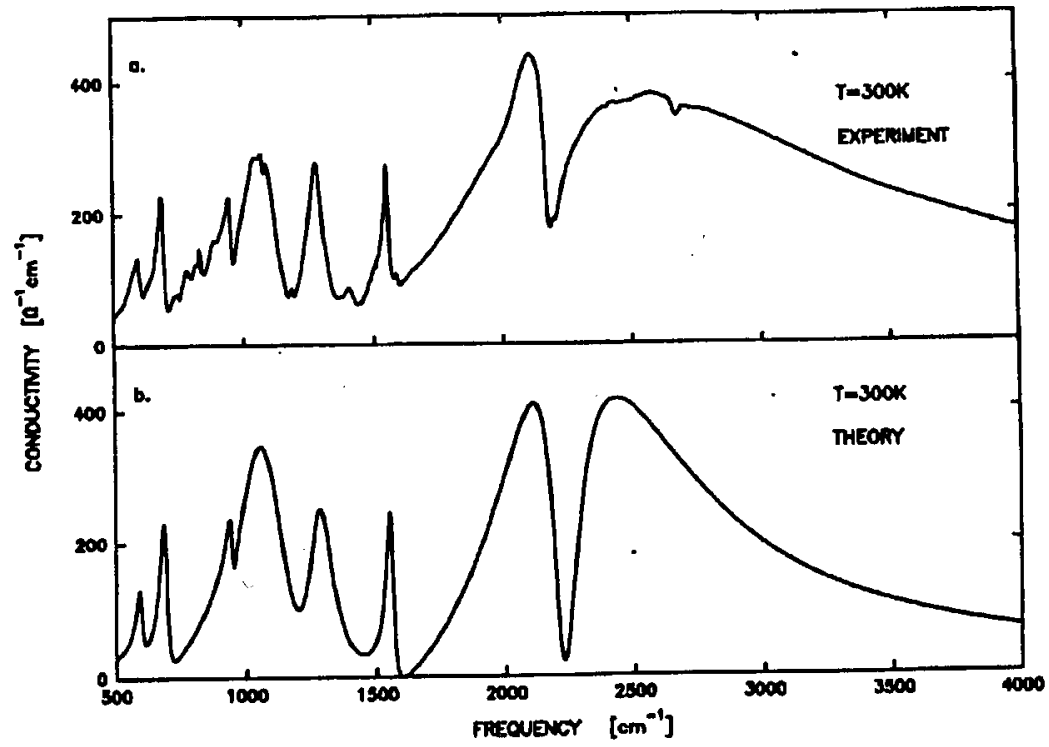

Fig. 1. Real part of the frequency dependent conductivity of TEA(TCNQ) 2 as deduced by dispersion analysis of the measured reflectivity at $300 \mathrm{~K}$ (a) and calculated theoretically (b) according to Eq. (3).

fit to the low temperature data too (Fig. 2), although it ought to be pointed out that the dimer model leaves out the doublet structure of the activated $a_{\mathrm{g}}$ modes; 
the values of the frequencies of $a_{\mathrm{g}}$ modes are well fitted, while the conductivities are distinctly smaller than the experimental values.

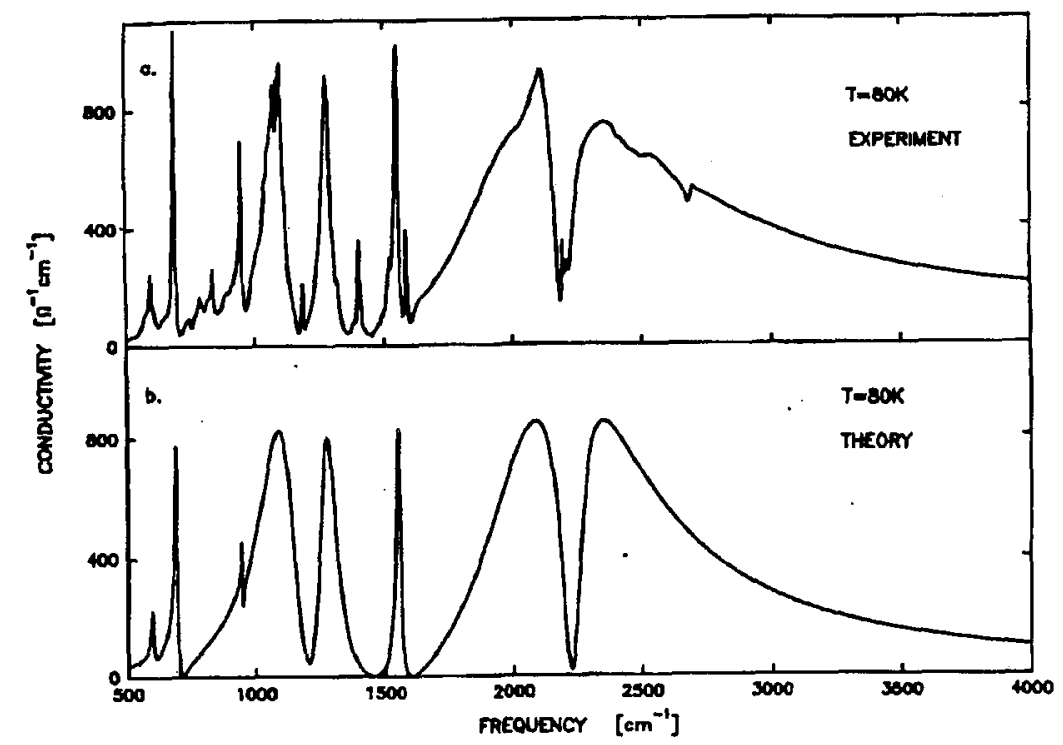

Fig. 2. Real part of the frequency dependent conductivity of TEA(TCNQ) 2 as deduced by dispersion analysis of the measured reflectivity at $80 \mathrm{~K}$ (a) and calculated theoretically (b) according to Eq. (3).

The calculated frequencies of the $a_{\mathrm{g}}$ modes are practically independent on temperature. On the other hand, one can observe a decrease in the CT band parameters, $\omega_{\mathrm{CT}}$ and $\Gamma_{\mathrm{CT}}$, with lowering of temperature.

The values of the coupling constants at $300 \mathrm{~K}$ are listed in Table I whereas the calculated frequencies and natural widths of activated $a_{\mathrm{g}}$ bands together with their experimental frequencies at $300 \mathrm{~K}$ are shown in Table II. The $g_{\alpha}$ values found by us can be compared with the $g_{\alpha}$ determined by Steigmeier et al. [7] and calculated by Lipari et al. [14]. The $g_{\alpha}$ values decrease monotonically when the temperature lowers. This result is strange because the coupling constants should not be temperature dependent unless the average molecular charge changes with temperature. It is not the case of TEA(TCNQ) $)_{2}$. One can expect some changes in the charge distribution, but not a change of the average molecular charge at the phase transition temperature. Nevertheless, we did not observe anomalies in the temperature dependence of the determined coupling constants $g_{\alpha}$. It seems that the apparent temperature dependence of the $g_{\alpha}$ constants is most likely an indication of the inadequacy of the dimer model. The role of the fitting procedure (search for the best correspondence of the calculated spectra to the experimental ones by fitting various parameters, among them the coupling constants) is also very important.

The theory of the dimer with one radical electron accounts not badly for the 
IR reflection in TEA(TCNQ) 2 at the high-temperature region, above the phase transition, of course if one neglects the doublet structure of the activated $a_{\mathrm{g}}$ modes. On the other hand, the spectral properties of the salt below the phase transition temperature are poorly described by the dimer model. This fact, in addition to the structural data [10], is an argument for the search for a better model describing the splitting of the $a_{\mathrm{g}}$ bands, in particular.

\section{TABLE I}

The fitted coupling constants of TEA(TCNQ $)_{2}$ found from the dimer model.

\begin{tabular}{c|c|c|c|c}
\hline \hline & $\omega_{\alpha}$ & \multicolumn{3}{|c}{$g_{\alpha}\left[\mathrm{cm}^{-1}\right]$} \\
\cline { 3 - 5 }$\alpha$ & {$\left[\mathrm{cm}^{-1}\right]$} & $300 \mathrm{~K}$ & $300 \mathrm{~K}$ & $300 \mathrm{~K}$ \\
& {$[13]$} & fitted & {$[7]$} & calc. [14] \\
\hline 2 & 2229 & 228 & 69 & 423 \\
3 & 1602 & 361 & 240 & 1057 \\
4 & 1454 & 427 & 294 & 392 \\
5 & 1207 & 216 & 144 & 229 \\
6 & 948 & 44 & 20 & 237 \\
7 & 711 & 149 & 87 & 263 \\
8 & 602 & 84 & 36 & 18
\end{tabular}

TABLE II

The calculated frequencies and natural widths of $a_{\mathrm{g}}$ bands together with their experimental frequencies found from the optical conductivity of TEA(TCNQ $)_{2}$ at $300 \mathrm{~K}$.

\begin{tabular}{c|c|c|c|c}
\hline \hline$\alpha$ & $\begin{array}{c}\omega_{\alpha}\left[\mathrm{cm}^{-1}\right] \\
{[13]}\end{array}$ & $\begin{array}{c}\omega_{\alpha}^{\prime}\left[\mathrm{cm}^{-1}\right] \\
\text { exper. }\end{array}$ & $\begin{array}{c}\omega_{\alpha}^{\prime}\left[\mathrm{cm}^{-1}\right] \\
\text { fitted }\end{array}$ & $\begin{array}{c}\gamma_{\alpha}\left[\mathrm{cm}^{-1}\right] \\
\text { fitted }\end{array}$ \\
\hline 2 & 2229 & 2112 & 2111 & 6 \\
3 & 1602 & 1552 & 1550 & 0.01 \\
4 & 1454 & 1280 & 1283 & 50 \\
5 & 1207 & 1070 & 1059 & 60 \\
6 & 948 & 948 & 938 & 15 \\
7 & 711 & 686 & 682 & 14 \\
8 & 602 & 594 & 588 & 20
\end{tabular}

The tetramer theory gives a better fit to the experimental data within the whole temperature range. It is shown for two selected temperatures $300 \mathrm{~K}$ and 


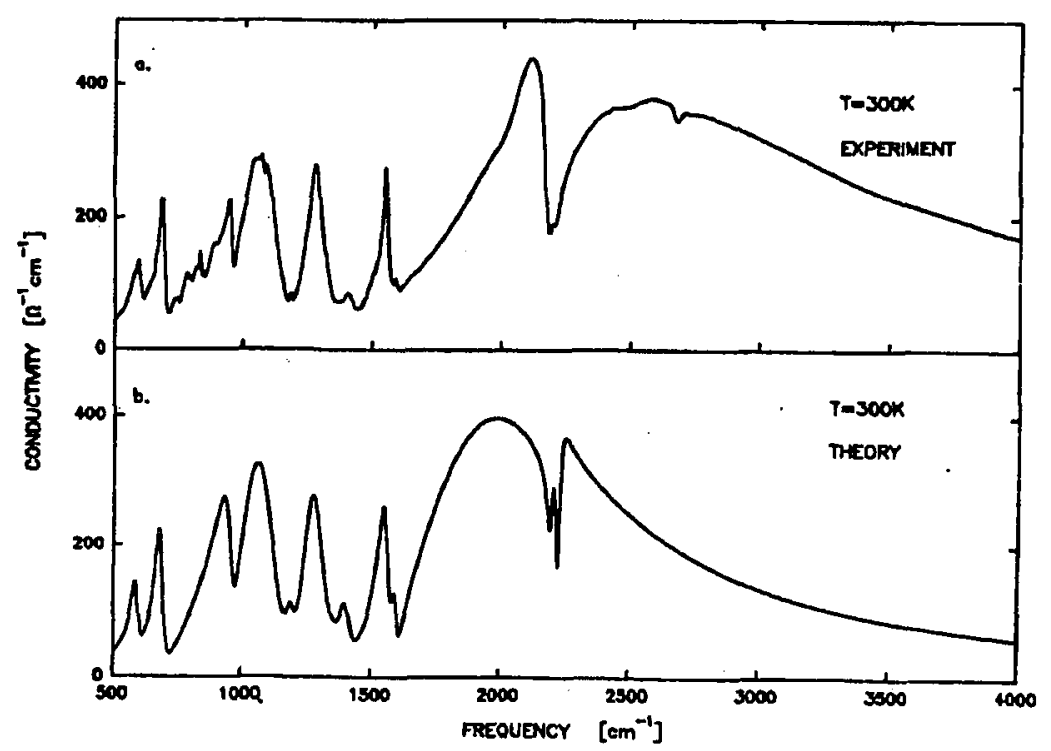

Fig. 3. Real part of the frequency dependent conductivity of TEA(TCNQ) 2 as deduced by dispersion analysis of the measured reflectivity at $300 \mathrm{~K} \mathrm{(a)} \mathrm{and} \mathrm{calculated}$ theoretically (b) according to Eq. (4).

$80 \mathrm{~K}$ in Figs. 3 and 4, respectively. The calculated values of the frequencies and conductivities of the activated $a_{\mathrm{g}}$ modes are in very good agreement with the experimental data within the whole temperature range. The tetramer model with two electrons per tetramer provides exactly the fine, doublet structure of the vibronic bands. The energies of both wide and narrow components are conformable to the experimental data with an accuracy better than $\pm 1 \%$. However the band $\omega_{2}$, which coincides with the CT band, is worse fitted (below $6 \%$ ). Peak values of the electrical conductivity deviate a little more from the experimental ones. $A$ choice of the lineshapes is the main source of these divergences. Nevertheless, the physical meaning lies in the integral intensity of the band and this is why the divergences in the maximal values of $\sigma(\omega)$ are not physically meaningful. The calculated spectral parameters $\Gamma_{\mathrm{CT}}, \omega_{\alpha}{ }^{\prime}, \omega_{\alpha}{ }^{\prime \prime}, \gamma_{\alpha}{ }^{\prime}, \gamma_{\alpha}{ }^{\prime \prime}$ do not show anomalies at the phase transition temperature. Similarly as for the dimer model fit we observe a decrease in the CT band parameter with lowering of temperature from $\Gamma_{\mathrm{CT}}(300 \mathrm{~K})=1319 \mathrm{~cm}^{-1}$.

The values of the coupling constants for both wide and narrow components of the doublets are collected in Table III. The values $g_{\alpha}{ }^{\prime}$ and $g_{\alpha}{ }^{\prime \prime}$ differ one from another and are not exactly the same as found from the dimer model. The differences between $g_{\alpha}{ }^{\prime}$ and $g_{\alpha}{ }^{\prime \prime}$ reflect the experimental data showing that the component shifts as well as intensities are different for various $a_{\mathrm{g}}$ modes. In spite of some divergences between $g_{\alpha}{ }^{\prime}$ and $g_{\alpha}{ }^{\prime \prime}$ the general tendency is kept. For some bands, e.g. $602,711,948$ and $1207 \mathrm{~cm}^{-1}$ the coupling constants are temperature independent within the fit accuracy. For the others one can observe an increase in $g_{\alpha}{ }^{\prime}$ and small decrease in $g_{\alpha}{ }^{\prime \prime}$ with lowering of temperature. The absence of a general ten- 


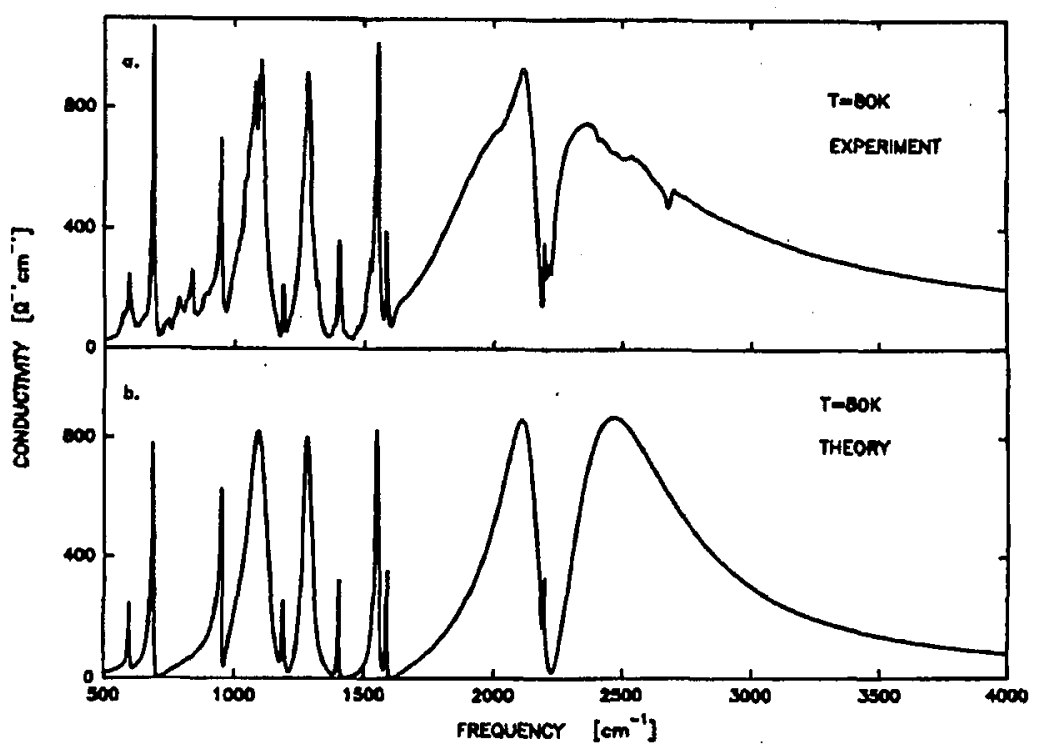

Fig. 4. Real part of the frequency dependent conductivity of TEA(TCNQ) ${ }_{2}$ as deduced by dispersion analysis of the measured reflectivity at $80 \mathrm{~K}$ (a) and calculated theoretically (b) according to Eq. (4).

TABLE III

The values of the coupling constants for both wide and narrow components of the $a_{\mathrm{g}}$ doublets.

\begin{tabular}{c|c|c|c|c}
\hline \hline \multirow{2}{*}{} & $\omega_{\alpha}\left[\mathrm{cm}^{-1}\right]$ & $g_{\alpha}^{\prime}\left[\mathrm{cm}^{-1}\right]$ & $g_{\alpha}^{\prime \prime}\left[\mathrm{cm}^{-1}\right]$ & $g_{\alpha}\left[\mathrm{cm}^{-1}\right]$ \\
& {$[13]$} & $\begin{array}{c}300 \mathrm{~K} \\
\text { fitted }\end{array}$ & $\begin{array}{c}300 \mathrm{~K} \\
\text { fitted }\end{array}$ & \\
\hline 2 & 2229 & 141 & 199 & 423 \\
3 & 1602 & 267 & 510 & 1057 \\
4 & 1454 & 474 & 486 & 392 \\
5 & 1207 & 249 & 300 & 229 \\
6 & 948 & - & 197 & 237 \\
7 & 711 & 220 & - & 263 \\
8 & 602 & 138 & - & 18
\end{tabular}

dency in the temperature dependence suggests that the tetramer model is good, as the first approximation to describe the TEA(TCNQ $)_{2}$ spectra in a wide temperature range. On the other hand the observed divergences show that the tetramer model with two electrons per tetramer does not describe perfectly all properties of the reflection spectra of TEA(TCNQ) $)_{2}$ salt. The essential source of the divergences results from the assumption that the tetramers are isolated - it is not the 
case of the salt under investigation. Unfortunately, it is very difficult to take into consideration the intertetramer interactions. Of course, the assumption of regular organization of the TCNQ tetramers is not also fulfilled in TEA(TCNQ) $)_{2}$ crystals.

The calculated frequencies and natural widths of the components of activated $a_{\mathrm{g}}$ bands at $300 \mathrm{~K}$ are shown in Table IV. The component shifts are correlated with the fitted electron-molecular vibration coupling constants.

\section{TABLE IV}

The calculated frequencies and natural widths of the components of activated $a_{\mathrm{g}}$ bands at $300 \mathrm{~K}$.

\begin{tabular}{c|c|c|c|c|c|c}
\hline \hline & $\begin{array}{c}\omega_{\alpha} \\
{\left[\mathrm{cm}^{-1}\right]} \\
{[13]}\end{array}$ & $\begin{array}{c}\omega_{\alpha}^{\prime} \\
{\left[\mathrm{cm}^{-1}\right]} \\
\text { exper. }\end{array}$ & $\begin{array}{c}\omega_{\alpha}^{\prime \prime} \\
{\left[\mathrm{cm}^{-1}\right]} \\
\text { exper. }\end{array}$ & $\begin{array}{c}\omega_{\alpha}^{\prime} \\
{\left[\mathrm{cm}^{-1}\right]} \\
\text { fitted }\end{array}$ & $\begin{array}{c}\omega_{\alpha}^{\prime \prime} \\
{\left[\mathrm{cm}^{-1}\right]} \\
\text { fitted }\end{array}$ & $\begin{array}{c}\gamma_{\alpha}^{\prime}=\gamma_{\alpha}^{\prime \prime} \\
{\left[\mathrm{cm}^{-1}\right]} \\
\text { fitted }\end{array}$ \\
\hline 2 & 2229 & 2112 & 2202 & 1984 & 2209 & 9 \\
3 & 1602 & 1552 & 1588 & 1549 & 1592 & 12 \\
4 & 1454 & 1280 & 1404 & 1279 & 1400 & 35 \\
5 & 1207 & 1070 & 1186 & 1062 & 1194 & 32 \\
6 & 948 & - & 948 & - & 934 & 25 \\
7 & 711 & 686 & - & 684 & - & 16 \\
8 & 602 & 594 & - & 586 & - & 23
\end{tabular}

\section{Conclusions}

The present study provides an extensive approach of the IR optical properties of TEA(TCNQ) $)_{2}$ salt in terms of the dimer model with one electron per dimer and of the tetramer model with two electrons per tetramer. The high- $T$ phase experimental data are satisfactorily described by the dimer model. The tetramer model provides the fine structure of the activated $a_{\mathrm{g}}$ bands and describes correctly the TEA(TCNQ) ${ }_{2}$ spectra within the wide temperature range, between 80 and $300 \mathrm{~K}$. The results suggest that the semiconductor-semiconductor phase transition at about $220 \mathrm{~K}$, which occurs mainly in the cation sublattice, is not connected with a reorganization of the electron distribution in the TCNQ stacks; both the dimer and tetramer fits do not show anomalies at the phase transition temperature.

The dimer model is not adequate for describing the low- $T$ phase of TEA(TCNQ $)_{2}$. On the contrary the tetramer model is more adequate for the interpretation of the IR spectra of TEA(TCNQ) $)_{2}$. However, the inter-tetramer interactions, difficult to be taken into consideration, are responsible for some divergences between experimental and calculated spectra. 


\section{Acknowledgments}

The authors wish to thank J.P. Farges and A. Brau for the delivery of single crystals of TEA(TCNQ) $)_{2}$. We acknowledge the financial support from the Committee for Scientific Research (grant 2020091 01)). We are grateful to R. Świetlik for many useful discussions.

\section{References}

[1] I. Olejniczak, A. Graja, Acta Phys. Pol. 83, 517 (1993).

[2] M.J. Rice, Solid State Commun. 31, 93 (1979).

[3] M.J. Rice, V.M. Yartsev, C.S. Jacobsen, Phys. Rev. B 21, 3437 (1980).

[4] V.M. Yartsev, R. Świetlik, Rev. Solid State Sci. 4, 69 (1990).

[5] V.M. Yartsev, Phys. Status Solidi B 126, 501 (1984).

[6] A. Brau, P. Brüesch, J.P. Farges, W. Hinz, D. Kuse, Phys. Status Solidi B 62, 615 (1974).

[7] E.F. Steigmeier, H. Auderset, D. Baeriswyl, M. Almeida, Mol. Cryst. Liq. Cryst. 120, 163 (1985).

[8] M.V. Belousov, A.M. Vainrub, R.M. Vlasova, Fiz. Tverd. Tela 18, 2637 (1976).

[9] J.P. Farges, J. Phys. (Paris) 46, 465 (1985).

[10] A. Filhol, M. Thomas, Acta Crystallogr. B 40, 44 (1984).

[11] V.M. Yartsev, M.J. Rice, Phys. Status Solidi B 100, K97 (1980).

[12] G. Janssen, R. Visser, H.Th. Jonkman, J. de Baer, J. Kommandeur, J. Phys. (Paris) Coll. 44, C3-1587 (1983).

[13] A. Girlando, C. Pecile, Spectrochim. Acta A 29, 1859 (1973).

[14] N.O. Lipari, C.B. Duke, R. Bozio, A. Girlando, C. Pecile, A. Padva, Chem. Phys. Lett. 44, 236 (1976). 